\title{
A Brief History of Neuronal Reconstruction
}

\author{
Stephen L. Senft
}

Published online: 24 February 2011

(C) Springer Science+Business Media, LLC 2011

The routes taken by peripheral nerves and the intricacy present even in the external features of the CNS had been understood in considerable detail at the time of Andreas Vesalius (1543) and Bartholomeo Eustatius (1552). But the astonishing underlying and microscopically symplectic character of CNS brain tissue only became evident in fitful stages and over the following 300 and 50 years. Today's opportunity to precisely reconstruct individual neurons and circuits, presently expressed in the DIADEM challenge, is based on a long sequence of conceptual and technological advances in our understanding of brain. A selection among these are highlighted in the following abbreviated historical account, which focuses first on our means of knowing that neurons exist, and then on how we learned to record and analyze their shapes.

\section{Brains to Neurons}

The hint that biological beings (and hence their brains) might be composed of subunits arose with Robert Hooke's 1660 's use of an early $40 \times$ microscope (Zacharias Janssen and his father Hans had made a $9 \times$ compound microscope circa 1595), which Hooke trained on miniscule objects that he could lay his hands on, such as cork. Its reticulated bark (and also the pith of other plants) reminded him of a hallway of 'cells' in which monks write manuscripts. Were he to have trained his optics on bits of brain - in that era

\section{S. L. Senft $(\square)$}

Krasnow Institute for Advanced Study, George Mason University, MS2A1, 4400 University Drive,

Fairfax, VA 22030-4444, USA

e-mail: sls@capecod.net neither stained nor fixed - it is likely that Hooke would have seen merely a glistening blur having a range of whitish and greyish tinges. In Italy, Marcello Malpighi was in fact, at that time, painstakingly examining brain under the microscope. He also boiled that tissue in water, or smeared it with ink, and by 1665 was thus able to discern fibers (which he interpreted as glandular) in spinal cord, fish optic nerve and corpus callosum, and in human white matter. Availed with even higher magnification, Antony van Leeuwenhoek peered at fresh cow optic nerve in 1674, and reported it to consist of threadlike chains of globules. By 1684 he also had inspected decomposing turkey brain, and had seen nerve profiles in cross sections, hand-cut and wetted in wine (and occasionally tinged using saffron).

Mankind's ability to discern microscopic features of brain beyond these beginnings required not only improved optics but better methods for preserving bodies and for generating tactile or visual contrast with which to differentiate minute components in tissue. It also required (and reinforced) a major evolution in cognitive perspective with respect to the natural outer and inner world. Reflex explanations in terms of vitalism (a force both ubiquitous and pan-functional) were gradually refined by discernments which taught that variation in function arises from differing mixtures of components and relationships.

Early advances in fixation began with Raymond Vieussens, who, circa 1684 , hardened human brain by boiling it in oil and thereby was able freehand to partially follow numerous fasciculi within the CNS. From the 1690's through the 1720's Frederik Ruysch used numerous compounds to embalm tissue, including brain. Trained as an apothecary, he is reported as having used alcohol and black pepper (liquor balsamicus); or potassium nitrate (saltpeter, potash) with hydrochloric or nitric acid (for nerve tracts). In addition he is said to have used clotted pig's blood, 
mercuric oxide and the newly synthesized Berlin (Prussian) Blue. That colorant, which binds heavy metals, was created by Johann Jacob Diesbach, in 1706, while trying to modify a naturally red, but very expensive, imported cochineal dye).

Certain macroscopic brain regions do have distinctive intrinsic textures or colors, (in fresh tissue, or by using some of the Ruysch methods) visible to the naked eye: the 'lineola albidior' border around V1, described (circa 17761782) from frozen human brain by Francesco Gennari, or the locus 'caeruleus', the substantia 'nigra', the 'red' nucleus and globus 'pallidus': all attentively described or named by Felix Vicq D'Azyr between 1786 and 1800.

However, the subtle aspects of brain anatomy only began to emerge through an analysis of texture (Marie Françiose Xavier Bichat famously enumerated many such 'tissues' by the end of the 1700s). By 1732 Alexander Monro (the first of three by this name) was able to characterize nerves microscopically as comprised of minute parallel (branching) threads. In 1779 Georgius Prochaska found that fresh brain CNS appeared to contain numerous "globules". In 1781 Felice Gaspar Ferdinand Fontana discovered that the threads seen by Munro were transparent uniform cylinders out of which could ooze plasm. Brain minutia of this sort became easier to study at leisure after 1809 , when Johann Christian Reil (who coined "psychiatry") systematically used alcohol (followed by potash and ammonia) to fix the brain. Too, micro-dissection was refined: when Charles Bell, Herbert Mayo and Richard Dugard Grainger inspected brain in the early decades of the 1800 s, they peeled the brain apart, tract by tract, discovering many of the macroscopic routes of communication within the CNS. In search of deeper understanding they teased the tissue apart with small needles, revealing central brain material to be a felt-like mat containing minute fibers whose start and end were, however, indeterminable.

In the late 1820s Jan Evangelista Purkinje began used a device (named a "microtome" by Charles Chavalier in 1839) for cutting thin brain sections. Similar machines had been in use 50 years earlier by George Adams for plant material, although it was not until 50 years later that Rudolf Jung and Rudolf Thoma made them commercially popular. Sections (including those made free-hand) allowed Purkinje to see through tissue using the microscope in transmitted light mode (the best by this time had achromatic lenses), and in 1837 he described his eponymous cells, although did not see their full arborization. His use of balsam to seal sections on slides further helped to prolong the duration of study of the prepared tissue. Prior to this era a 'slide' meant a holder made of bone having circular holes, and specimens were mounted between sheets of clear mica. By 1837 Joseph Jackson Lister's microscope objectives had become sophisticated enough to require a specific range of cover glass thickness (theoretically recognized by Giovanni Battista Amici in 1827). However, coverslips were not made to very high tolerance and thus their suitability was evaluated individually using a micrometer. Today's ubiquitous 1 by 3 inch glass slide was standardized by the Royal Microscopy Society soon afterwards, in 1840.

By 1833 Christian Gottfried Ehrenberg, using unfixed and unstained material, had distinguished fibers in peripheral nerve, and transparent globules in ganglia and cerebral cortex which he thought might have been exudates from blood (presumed to be more 'vital'). Purkinje's student Gabriel Gustav Valentin by 1836 was able to resolve individual brain cells and some of their substructures, including the nucleus (seen first in non-brain tissue by Franz Bauer in 1802, and named in 1831 by Robert Brown, of "Brownian" motion), its nucleoli, and the initial portion of a uniform cylindrical process. In 1840 Jules Gabriel François Baillarger reported that (thin-sectioned) cerebral cortex examined by eye consists of six layers (stacked "like a galvanic pile"), and that fibers connected this gray matter to the underlying white.

Soon the means of fixation were extended with a variety of additional chemicals. Vinegar, copper sulfate, chromic acid were used by Adolphe Hannover by 1840, and by Purkinje, and Johannes Peter Müller added potassium dichromate (in combination with sodium sulphate this is "Müller's fluid"). Similar fixatives enabled Robert Remak to discern the presence of membrane delimiting cell bodies and, in studying peripheral nerves to their source, he was able to associatively link cells to their processes by 1838 about the time that Theodor Schwann was establishing the general cell theory as a principle (presaged by Rene Joachim Henri Dutrochet in 1824).

By 1842, Benedikt Stilling sufficiently refined the microtome to enable him to make serial sections and to better appreciate the three dimensional aspects of spinal cord organization. In the CNS, Remak was able to distinguish myelinated fibers by their greyish color, and in 1844 showed fibers infiltrating and coursing through cerebral cortex. A major advance came in 1852 when Rudolf Albert von Koelliker, using cerebral cortex treated with chromic acid, described pyriform and fusiform cells (later categorized systematically by shape and location by Rudolf Berlin and Theodor Meynert) out of which he could see microscopic branching processes ending in minute fibrils. Von Koelliker also noted that straight fibers traveling through grey matter could possess varicosities.

The ability to discern neural structure again vastly improved when it became possible to differentially stain parts of or types of cells. In the 1850s Joseph Von Gerlach and then Alfonso Corti used the natural substance carmine (from the cochineal beetle) to stain brain, whereby occasional individual fibers could now be 
resolved beautifully, although still appearing intermingled en masse. Likewise by 1863 , picric acid and hematoxylin (from tropical logwood, long used to dye paper and cloth) were used as stains, and acridine (from coal tar) followed in the 1870 s. In this mid-Industrial age, the desire to mass-produce brightly colored clothes began to be served by the synthetic dye industry, starting with aniline, created by William Henry Perkin, in 1856at age 18, and while trying to create quinine! This soon led to an enormous range of tissue stains (aniline blue, methylene blue, fluorescein, eosin, basic fuchsin), all tested against brain, and with fascinating results in staining various portions of its constituents. The remarkable ability of osmium to fix and tinge tissue began to be used in the CNS, after its effect was noted by (circa 1865) by Max Johann Sigismund Schultze - as he was attempting to force fireflies to light up!

Using chromic acid and carmine, Otto Friedrich Karl Deiters was able to see, and dissect out, spinal motoneurons, complete with nucleus, protoplasmic extensions, and an initial "axis cylinder", in 1865 . It began to appear that these cells were connected in networks. From the work of Emil Heinrich du Bois-Raymond (1849) it became plausible (especially to Gerlach) that electrical impulses, known to exist in live tissue since the time of Luigi Galvani (c. 1780), might travel along these filamentous structures.

A several decades-old photographic industry, especially through its use of gum bichromate and silver nitrate, may have spurred Camillo Golgi's 1873 famous discovery that in brain similar compounds could form the "black reaction", revealing a subset of brain cells (selected by a still mysterious process) as dark spheroids connected to elaborately branching and intertwining filaments - apparently stained in their entirety. This new ability to see extensive arbors from thousands of individual cells was transformative for neuroanatomy and became an indispensable technique. Even though by exact criteria few cells could be proven to impregnate completely, this drawback could be viewed merely as a statistical issue (enough partial examples should give an accurate sense of the underlying type), and the method's limitations paled in comparison to its utility for revealing the distribution and shapes of numerous classes of brain cells. Not long afterward, in 1874, Vladimir Alekseyevich Betz processed the brains of many animal species with similar iodine/potassium chromate methods, as well as with carmine, and was able to describe his famous (nests of) giant pyramidal cells in human cortex. Karl Weigert contributed methods for staining myelin, starting in 1885 .

Four years later, the cell doctrine was elegantly elaborated for brain tissue as well, when with a trove of elegant histological preparations and through subtle reasoning Santiago Ramón y Cajal convincingly spearheaded the view (correctly, as we also now know from electron microscopy, tissue culture and a convergence of ancillary biochemical evidence) that nerve cells are independent entities, and do not form syncytia. His work relied heavily on his many modifications of the Golgi reaction, and on careful use of the latest microscope optics. As a result of studying a vast number of well stained examples from a variety of species he was able to deduce the functional principle of integrative flow in the brain: inward along widening converging branches, across the cell body, outward along longer fibers of uniform diameter and finally into a terminal arborization, at which point follower cells are stimulated and the process propagates, in a potentially endless chain.

At this point in history, the fundamental structural decomposition of brain into signal-passing networks of neurons embedded in a sea of glia and fenestrated by blood vessels was basically recognized in its present-day sense. [Modern histologists take note: this was all before Ferdinand Blum in 1893 reported using the antiseptic formaldehyde as a fixative!] The era's ability to study brain cells and their arbors in such detail gave rise to numerous terms and conceptualizations that are still in use today. In 1889 Wilhelm His coined the term dendrite, while in 1891 Heinrich Wilhelm Gottfried von Waldeyer-Hartz coined the term neurone. Five years later, in 1896, von Kolliker created the term axon, and the next year Charles Scott Sherrington coined the "clasping" term synapse. Indeed, these named concepts make possible the DIADEM challenge itself (which, through it's impetus to abstract histology into mathematical form, could contribute to the historical progression of explicating opaque brain functions in terms of components, by enabling such functions to be computable from precisely characterized named network elements).

\section{Neurons to Voxels}

It is one thing to view neurons and their dendrites and axons through a microscope - actually, with some tissue preparations that can be a stupefyingly marvelous experience, unequalled in it's impact on one's psyche, to this day, by any of the artful and increasingly sophisticated reconstructive methods discussed below-it is quite another to accurately measure their properties or to communicate them to other minds at distant places or times. Perhaps reconstruction techniques of the future will become comprehensive enough to mirror in quantitative (and thereby additionally profound) form the exquisiteness of what can be apprehended directly from well-processed tissue - and, eventually, to provide views even crisper than can be seen through an ocular. The history of the past century of reconstruction methods, sketched next, can be 
seen as a series of efforts cooperating towards that stilldistant goal.

At first, free-hand drawings were used (having long been made since the time of Leeuwenhoek, and he had them made for him) for recording microscopic views. These were often quite beautifully executed, yet could be a synthetic amalgam of visual impressions rather than a precise record of an individual preparation.

The camera lucida device used by artists such as William Hyde Wollaston ${ }^{1}$, in 1811 , was adapted to the microscope early on and made such records more veridical by providing a means for superposing the tracing with the view through the microscope. Shortly after mankind learned to paint with light, glass and chemistry, the first photomicrographic documentation was made (circa 1855).

However, reconstruction - the blending of all parts of a single cell into one view-was easier done with the camera lucida, because the depth of field could be compiled into a drawing more readily than at first could be photographed onto a single plate. Nonetheless, the natural desire to capture microscopic objects in at least three dimensions has generated (in a many attempts spanning more than a century) a great variety of inventive, albeit partial, reconstructive solutions.

By transferring the outlines of a series of drawings of an object to a sequence of wax cut-outs, early anatomists were able to make opaque 3D wax models of microscopic structures, such as done with human embryos by $\mathrm{His}^{2}$ in 1880, and by Gustav Born ${ }^{3}$ (physicist Max Born's father) in 1883. One drawback was that alignment of the physical sections was imperfect, even when external fiducials were added, by Nikolai Feofanovich Kastschenko ${ }^{4}$, in 1886.

The new availability of acetate film in movie format provided another medium for assembling 3D material. In 1907 Karl Reicher $^{5}$ took photographs of manually aligned sections to create films over 1000 sections in length. This was done at the scale of the whole human brain, and permitted one to reconstruct a dramatic sense of moving back and forth within the CNS. In 1927 P. F. Saxl ${ }^{6}$ recorded optical sections directly from the microscope to film to achieve similar effects at the microscopic level. In 1932

\footnotetext{
${ }^{1}$ Wollaston, W. H. (1811). Phil. Trans. R. Soc. Lond. 101, 96-105.

${ }^{2}$ His, W. (1880) Anatomie menschlicher Embryonen, F. C. W. Vogel, Leipzig Vol 1, pp 7-12.

${ }^{3}$ Born, G. (1883) Die Plattenmodellirmethode. Arch. f. Mikr. Anat., 22, 584-599.

${ }^{4}$ Kastschenko, N. (1886) Methode zur genauen Rekonstruktion kleinerer makroskopischer Gegenstande, Arch. Anat. Physiol. Abt., pp. 388-394.

${ }_{5}$ Reicher, K. (1907) Die Kinematographie in der Neurologic. Neurologisches Zentralblatt, 24, 496.

${ }^{6}$ Saxl (1927) Photogr. Korresp. 63.
}

Phillip R. Peacock and L. Woodhouse Price ${ }^{7}$ were able to image aligned series of sections onto $16 \mathrm{~mm}$ film (circa 50 lines/mm resolution) by projecting microscope images onto a screen, aligning them with respect to tracings of prior sections, and filming the result.

From 1946 to 1951 Erling S. Hegre and Alton D. Brashear $^{8}$ employed a variant of physical serial sectioning in which, at each cut of the microtome, the block face was stained and photographed. They also constructed a chinoperated focus device to help with this repetitive process! (This technique of serial block face imaging was given an EM context in 1981 by Stephen B. Leighton ${ }^{9}$ ).

In 1952 Vannevar Bush and Richard E. Hewitt ${ }^{10}$ [following Victor Widakowich, in $1907^{11}$ ] transferred actual sections (frozen) onto coated (and stained) acetate (as recently done more systematically onto mylar for electron microscopy, by Jeff W. Lichtman's ATLUM machines in $2006^{12}$ ).

In the 1950s morphometrics, which Meynert in 1872, and Betz in 1874, had pioneered for somatic shape, began to be developed for neurites. Most prominently Donald A. Sholl from $1953^{13}$ introduced diagrammatic methods, still in use today, for statistically deducing cortical dendritic packing density for and estimating numbers of potential dendritic interactions. Additionally, Siegfried Thomas Bok in $1959^{14}$ (and recapitulating his work begun in 1936) used pyramidal cell stylization [an emphasis on node points] as an aid for semi-quantitative comparison of these neurons across species.

Also at this time, the effects of arbor shape on the dynamics of signal spread began to be investigated mathematically. Wilfred Rall applied the (transatlantic) cable equations to neurites, both as reduced geometries and as full arbors, starting in $1959^{15}$. This made explicit the extent of steady state electotonus expected in a neuron of a given geometry. He later extended his analyses to include

\footnotetext{
${ }^{7}$ Peacock, P. R. and Price, L. W. (1932) On the cinematographic examination of serial sections as an aid to histology. Journal of the Royal Microscopical Society 52, 265-268.

${ }^{8}$ Hegre, E. S. and Brashear, A. D. (1946) Block-surface staining. Stain Technol. 21(4), 161-164.

${ }^{9}$ Leighton, S. B. (1981) SEM images of block faces, cut by a miniature microtome within the SEM-A technical note. Scan Electron Microsc. 2, 73-76.

${ }^{10}$ Bush V. and Hewitt, R. E. (1952) Frozen sectioning; a new and rapid method. Am. J. Pathol. 28(5), 863-873.

${ }^{11}$ Widakowich (1907) Zentralbl. Physiol. 21, 784.

${ }^{12}$ Hayworth, K.J., Kasthuri, N., Schalek, R. and Lichtman, J. W. (2006). Automating the Collection of Ultrathin Serial Sections for Large Volume TEM Reconstructions. Microscopy and Microanalysis, $12,86-87$

${ }^{13}$ Sholl, D. A. (1953) Dendritic organization in the neurons of the visual and motor cortices of the cat. J. Anat. 87, pp. 387-406.

${ }^{14}$ Bok, S. T. (1959) Histonomy of the Cerebral Cortex. Wiley, New York.

${ }^{15}$ Rall W. (1959) Branching dendritic trees and motoneuron membrane resistivity. Exp. Neurol. 1, 491-527.
} 
transients, so that the full dynamics that Alan Lloyd Hodgkin and Andrew Fielding Huxley had established in squid axon in 1952 could (in principle) be evaluated for entire arbors, if they were known accurately enough. In 1962 E. Ramon-Molinar ${ }^{16}{ }^{\text {attempted to categorize dendrites }}$ using shape criteria, hoping to learn whether their structure was systematically sculpted by functional constraints. These open questions helped to fuel the desire for additional and improved reconstruction of neurons in 3D.

The 1960's was also the era when it first became feasible to incorporate the computer into the process of tracing, archiving, and analyzing neuronal arbors. The earliest to do so were Edmund M. Glaser and Hendrik van der Loos (1962-1965) ${ }^{17}$. They employed an analog computer to read the encoded locations of stage and fine focus as they manually traced neurons. The resulting $3 \mathrm{D}$ vectors were shown on a CRT screen, but could only be saved as printed 2D orthographic projections.

Several purely optical methods were devised that facilitated the reconstruction process. Martin H. Zimmerman and Barry P. Tomlinson made an optical shuttle to connect two microscopes with a beam splitter in $1966^{18}$. Interleaved physical sections could be aligned, by rotating and translating each stage (misalignment could be made to shimmer if illumination was made to alternate), and the result photographed. In 1967 Roger Lannes de Montebello invented $^{19}$ an extremely innovative and truly 3D display for film loops, in which an image of the reconstructed object was made to hover in the air.

Computer-based systems rapidly evolved. Philip G. Stein, in $1968^{20}$, was able to electromechanically translate the stage in $0.6 \mathrm{mu}$ increments and step in z by $0.2 \mathrm{mu}$, while tracing. By saving a stack of untraversed branch points, when an endpoint was manually entered the field of view could be made return to the most recent pending location. Moreover, the system could digitize an image of the microscope view with an XY scanning photomultiplier. This seeded the idea

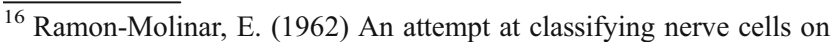
the basis of their dendritic patterns, J. Comp. Neurol., 119, 211-227.

${ }^{17}$ Glaser, E.M. and Van der Loos, H. (1965) A semi-automatic computer microscope for the analysis of neuronal morphology. IEEE Trans. Biomed. Eng. BME12 (1), 22-31.

${ }^{18}$ Zimmermann, M. H. and Tomlinson, P. B. (1966) Analysis of complex vascular systems in plants: optical shuttle method. Science $152,72-73$.

${ }^{19}$ De Montebello, R. L. (1969) The RLM Synthalizer technique and instrumentation for optical reconstruction and dissection of structures in three dimensions. Annals of the New York Academy of Sciences, $157,487-496$.

${ }^{20}$ Stein, P. G., Lipkin, L. E. and Shapiro, H. M. (1968) Spectre II: General-Purpose Microscope Input for a Computer. Science 166, 328333.

${ }^{21}$ Selverston, A. I. and Kennedy, D. (1969) Structure and function of identified nerve cells in the crayfish. Endeavour 28, 107-113.
}

for computationally analyzing images in order to automatically trace in XYZ. Allen I. Selverston ${ }^{21}$ used a PDP 11 and Vector General display in 1969 to reconstruct filled cells fragmented by sectioning. Sections were aligned in computer memory and the user traced the center of the contours to produce a tree. The important novel aim in this work was to acquire data (including accurate diameters) suited for mathematical modeling of electrotonus in stomatogastric ganglion neurons.

The manual method of Glaser and van der Loos for direct tracing 'on-line' (with a cursor visible in the microscope image) gave rise to a host of semi-automatic systems whose authors initially had aimed for fully automatically arbor-tracing methods. An important effort was that of M. Weinstein, Kenneth R. Castleman and Henry Fuchs at JPL (1971-74) $)^{22}$, who were able to visualize 3D stacks of optical sections (1000 by 1000 digitized) in stereo, with simple methods for deblurring and edge detection. The data traced from these stacks was output as abstracted tree structures. Other notable attempts include those by Catherine Fitzgerald Garvey in $1972^{23}$, who tried to get a "vidisector' to automatically follow edges in TV images taken from the microscope, and that of D. Raj Reddy, who by $1973^{24}$ likewise digitized optical sections of intracellularly stained neurons at 1000 by 1000 pixels and then thresholded the images for the purpose of algorithmically extracting series of profiles. (A very modern approach, but implemented on a PDP/10 having a then-vast $192 \mathrm{~K}$ of memory!)

The semi-automatic strategy is epitomized by a system for arbor tracing of Golgi-impregnated material (single sections), using a PDP/12 connected to a Zeiss Universal microscope, made by Donald F. Wann et al., in $1973^{25}$. In 1975 Rodolfo R. Llinás and Dean E. Hillman $^{26}$ also constructed a semi-automatic tracing system, based on vectors. By 1977 they superimposed live and stored images for alignment and image analysis.

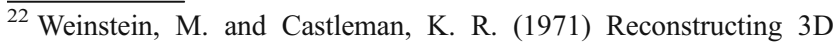
specimens from 2D section images. Proc. Soc. Photo-opt. Inst. Eng. 26, 131-138.

${ }^{23}$ Garvey, C. F., Young, J. H. Jr., Coleman, P. D. and Simon, W. (1973) Automated three-dimensional dendrite tracking system. Electroencephalogr. Clin. Neurophysiol. 35 (2), 199-204.

${ }^{24}$ Reddy, D. R., Davis, W. J., Ohlander, R. B. and Bihary, D. J. (1973) "Computer Analysis of Neuronal Structure" in Intracellular Staining in Neurobiology, S. B. Kater and C. Nicholson, Eds. Springer-Verlag, New York, pp. 227-253.

${ }^{25}$ Wann, D. F., Woolsey, T. A., Dierker M. L. and Cowan, W.M. (1973) An on-line digital-computer system for the semiautomatic analysis of Golgi-impregnated neurons. IEEE Transactions on Biomedical Engineering BME20 (4), 233-247.

${ }^{26}$ Llinas, R. and Hillman, D.E. (1975) A multipurpose tridimensional reconstruction computer system for neuroanatomy. In: M. Santini, Editor, Golgi Centennial Symposium Proceedings, Raven Press, New York pp 71-79.
} 
Albert Michael Paldino and Erich M. Harth in $1977^{27}$ addressed the problem of merging multiple section files. Likewise Robert D. Lindsay by $1977^{28}$ had a multisection device with operator-assisted optimization of alignment, running on a PDP/8 and with a Graf-Pen (sonic digitizer) placed over the CRT screen for tracing. Michael L. Dierker, circa 1980, added provisions for truncated and obscured data point types to the now multisection multi-cell Wann system. Particular attention continued to be placed on compensating for a series of artifacts, including tissue shrinkage and distortions introduced by the microscope optics. These issues were reviewed by Timothy J. DeVoogd in $1981^{29}$.

At this point in time the impetus to reconstruct neurons came equally from light microscopy (which aimed to reconstruct a tree of line segments) and from electron microscopy which primarily aimed to reconstruct a tree of profiles (see the work of Fritiof F. Sjostrand, circa $1958^{30}$ ). As a result, several computer-equipped laboratories created hybrid methods which could do both. For instance, Cyrus Levinthal and Randle Ware, in 1971-1972 ${ }^{31}$, aligned successive EM negatives via a pellicle (beam-splitter). By alternating illumination they could visually highlight misalignments. The aligned negatives were photo-transfered to cine film. Using an Adage Graphic Terminal for display, tracings (of Daphnia and Rotifer) were made as boundaries or as arbor-like vectors (via pushdown stack), with 'end', 'branch', 'continuation' and 'synapse' data-types for topological tree abstraction. The extensive Daphnia work was summarized by Eduardo Macagno in $1979^{32}$. Later this approach was continued at Cal Tech, with a PDP 11/45, for C. elegans. Also stemming from this source was an elegant long term EM reconstruction project for retina (currently still active) carried out by Peter Sterling ${ }^{33}$. Additional

\footnotetext{
${ }^{27}$ Paldino, A. M. and Harth, E. (1977), Some quantitative results on Golgi impregnated axons in rat visual cortex using a computer assisted video digitizer. The Journal of Comparative Neurology, 176, 247-261. ${ }^{28}$ Lindsay, R. D. (1977) "The Video Computer Microscope and ARGOS," in Computer Analysis of Neuronal Structures. R. D. Lindsay, Ed. Plenum, New York, pp.1-20.

${ }^{29}$ DeVoogd, T. J., Chang, F.-L.F., Floeter, M.K., Jencius, M.J. and Greenough, W.T.,(1981) Distortions induced in neuronal quantification by camera lucida analysis: Comparisons using a semi-automated data acquisition system. Journal of Neuroscience Methods 3 (3), 285294.

${ }^{30}$ Sjostrand F. S. (1958). Ultrastructure of retinal rod synapses of the guinea pig eye as revealed by three-dimensional reconstructions from serial sections. J. Ultrastructure Res. 2, 122-170.

${ }^{31}$ Levinthal C. and Ware, R. (1972) Three dimensional reconstruction from serial sections. Nature 236, 207.

32 Macagno, E. R., Levinthal, C. and Sobel, I. (1979) Threedimensional computer reconstruction of neurons and neuron assemblies, Ann. Rev. Biophys. Bioeng. 8, 323-351.

${ }^{33}$ Stevens, J. K., McGuire, B. A. and Sterling, P. (1980) Toward a functional architecture of the retina: serial reconstruction of adjacent ganglion cells. Science, 207 (4428), 317-319.
}

reconstructive principles have been developed for EM: David J. De Rosier and Aaron Klug, $1968^{34}$, performed EM reconstructions of subcellular structures along the lines of $\mathrm{X}$-ray diffraction. Later, Lee Peachey ${ }^{35}$ explored tissue reconstruction by tomographic means. These methods have modern optical homologues.

[See Randle W. Ware and Vincent Lopresti, $1975^{36}$, for a comprehensive history of the EM side of efforts to reconstruct brain tissue, and see Michael L. Dierker, $1980^{37}$, and Joseph J. Capowski, $1985^{38}$, for a history of the light-based methods.]

The state of development (including numerous efforts not listed here) was exciting enough that in 1979 Macagno could optimistically speak for the field: "The simplest neuroanatomical problem to which computer pattern recognition can be applied successfully with current techniques is that of determining the detailed anatomy of single dye-filled neurons." He cites Paul D. Coleman ${ }^{39}$, Hillman ${ }^{40}$, and others, who at the time primarily used simple thresholding methods.

As accurately traced neuron structural details became more commonplace, numerous other types of analysis were applied to neurites. Mathematical analyses of pyramidal cell branching in 3D, which extended the work of Bok, were performed in 1974 by Robert D. Lindsay and Arnold B. Scheibel $^{41}$. From the mid 1970s T. Hollingworth, M. Berry $^{42}$, and Harry B. M. Uylings, G. J. Smit ${ }^{43}$, and many

\footnotetext{
${ }^{34}$ De Rosier, D. J. and Klug, A. (1968) Reconstruction of Three Dimensional Structures from Electron Micrographs. Nature 217, 130 134.

${ }^{35}$ Peachey, L. D. and Heath, J. P. (1989) Reconstruction from stereo and multiple tilt electron microscope images of thick sections of embedded biological specimens using computer graphics methods, Journal of Microscopy 153, 193-204.

${ }^{36}$ Ware, R. W. and LoPresti, V. (1975) Three-Dimensional Reconstruction from Serial Sections. Int. Rev. Cytol. 40, 325-440.

${ }^{37}$ Dierker, M. L. (1980) Three Dimensional Reconstruction of neurons. (Thesis) Washington University, St. Louis, Missouri.

${ }^{38}$ Capowski, J. J. (1985) The reconstruction, display, and analysis, of neuronal structure using a computer. In R.R. Mize (ed.) The microcomputer in cell and neurobiology research. Elsevier, pp 85-109.

${ }^{39}$ Coleman, P. D., Garvey, C. F., Young, J. H. and Simon, W. (1977) Semiautomatic Tracking of Neuronal Process. In: R.D. Lindsay, Editor, Computer Analysis of Neuronal Structures pp 91-109. Plenum Press, New York.

${ }^{40}$ Hillman, D. E., Llinás, R. and Chujo, M. (1977) Automatic and semiautomatic analysis of nervous system structure. In: R.D. Lindsay, Editor, Computer Analysis of Neuronal Structures pp 73-90. Plenum Press, New York.

${ }^{41}$ Lindsay, R. D. and A. B. Scheibel. (1974) Quantitative analysis of the dendritic branching pattern of small pyramidal cells from adult rat somesthetic and visual cortex. Experimental Neurology 45, 424-434. ${ }^{42}$ Hollingworth T. and Berry, M. (1975) Network analysis of dendritic fields of pyramidal cells in neocortex and Purkinje cells in the cerebellum of the rat. Phil. Trans. R. Soc. Lond. B Biol. Sci. 8;270 (906), 227-64.

${ }^{43}$ Uylings, H. B., Smit, G. J. and Veltman, W. A. (1975) Ordering methods in quantitative analysis of branching structures of dendritic trees. Advances in Neurology 12, 248-254.
} 
other groups provided elegant combinatorial analyses of dendritic morphology, aimed at comparing arbors and at deducing neuritic growth modes. To aid this work, ordering systems were adopted from other fields (centripetal, counting from tips to root-such as Arthur Newell Strahler had used in $1957^{44}$ for river systems; or centrifugal: from stem to tip). A comprehensive assessment of neuronal form as represented by computer-aided reconstruction was given by Hillman in $1979^{45}$.

By the early 1980's semi-automatic methods became sufficiently codified to be commercialized. An early example was Joe Capowski's "Eutectics" system ${ }^{46}$ in which the operator 'drove' around in 3D, using a stage 25 by $75 \mathrm{~mm}$ in size and a z-encoder able to follow up to $2 \mathrm{~mm}$, keeping the traced neurite within a variable diameter circle fixed at the center of the field of view (for up to 1000 sample points per section). The ensemble of tracings could be merged across sections (taking perhaps 40 hours to reconstruct a cat motoneuron). Several innovations (from 1981) are worth noting: 'continuation' points were added automatically by the PDP $11 / 45$ computer as the operator tracked a neurite, and the sampling process was partially driven by means of voice recognition. Additional automation was possible by 1983. In the mid 1990s this product became supplanted in the market by the "Neurolucida" system from MicroBrightfield (founded in 1987 by $\mathrm{Ed}$ Glaser).

With a greater dissemination in the academic community made possible by commercialization it became desirable to standardize on the format of arbor data abstraction schemes. The multiplicity of formats in the early 1990 s, used by Capowski, Gwenn A. Jacobs and Rocky H.W. Nevin ${ }^{47}$, Dennis Alan Turner ${ }^{48}$ and James M. Bower and David Beeman ${ }^{49}$ gradually converged on the Southampton ('swc') format from Robert C. Cannon ${ }^{50}$. (Recently, attempts are

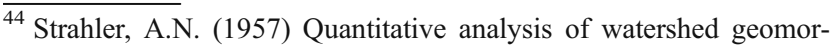
phology. Trans, Am. Geophys. Un. 38, 913-920.

${ }^{45}$ Hillman, D. E. (1979) Neuronal shape parameters and substructures as a basis of neuronal form. In Neurosciences: Fourth Study Program, pp 477-498, F. O. Schmitt and F. G. Worden, Eds.

${ }^{46}$ Capowski, J. J. and Sedivec, M. J. (1981) Accurate computer reconstruction and graphics of complex neurons utilizing state-of-theart interactive techniques. Computers and Biomedical Research 14, 518-532.

${ }^{47}$ Jacobs, G. A. and Nevin, R. (1991) Anatomical relationships between sensory afferent arborizations in the cricket cercal system. Anat. Rec. 231, 563-572.

${ }^{48}$ Turner D. A. (1984) Segmental cable evaluation of somatic transients in hippocampal neurons (CA1, CA3, and dentate). Biophys J 46, 73-84.

${ }^{49}$ Bower, J. M. and Beeman, D. (1994) The book of GENESIS: exploring realistic neural models with the GEneral NEural Simulation System. Springer-Verlag/ TELOS, New York. 409 pp.

${ }^{50}$ Cannon, R. C., Turner, D. A., Papyali, G., and Wheal, H. V. (1998) An on-line archive of reconstructed hippocampal neurons. Journal of Neuroscience Methods, 84(1-2), 49-54.
}

underway to systematically reconcile and extend the data formats used in reconstruction. See for instance the work of Sharon Crook, et al. in $2007^{51}$ ).

The Neurolucida system remains the most widely used semi-automatic system for operator tracing directly from the light microscope. In the last few years, it has been augmented to save series of optical sections and then to allow analysis off-line from the microscope. Because of this flexibility of input format, it is capable of working with one of the currently common main sources of neuron data: confocal image stacks.

With the advent of commercially marketed laser scanning confocal microscopes (such as Peter Wallén's Sarastro in $1988)^{52}$, it became possible to image fluorescent tissue as high resolution optical sections and to store the results as 3D data sets. Although the machines could make scans of up to 1024 by 1024 pixels, initially $64 \times 64 \times 648$-bit [0.25 MB] voxel data sets were considered achievements, because they conveyed a sense of isotropic volume and could be rotated in near real-time on the screen of a early workstation (such as made by Sun or Silicon Graphics). Data were archived onto floppy disks or DAT tape. Rapidly, $512 \times 512 \times \mathrm{N}$ in two or three wavelength-specific channels became the sweet spot, given the improved computer capacities of the time. Later $2048 \times 2048 \times N$, and 16-bit became common. Currently many gigabytes of data, often tiled in two and three dimensions (or even time varying), and having numerous channels, can be obtained by confocal and related methods. (See for instance the scans produced since 2007 by Kristina D. Micheva and Stephen J. Smith) ${ }^{53}$.

The bottleneck to reconstruction from each epoch has been processing speed, data access (whether data can be stored entirely in RAM or must be paged in from disk) and, most importantly, processing intelligence-for the longanticipated aim of expert automated image analysis proved elusive to implement. A host of image processing methods have been tried. The most common consisted of spatial filtering to suppress noise or to enhance edges, followed by global or local thresholding applied to the entire volume. In 1993 Fred W. Prior ${ }^{54}$ reported some success in automatic

\footnotetext{
${ }^{51}$ Crook, S., Gleeson, P., Howell, F., Svitak, J. and Silver, R. A. (2007) MorphML: Level 1 of the NeuroML Standards for Neuronal Morphology Data and Model Specification. Neuroinformatics 5 (2), 96-104.

${ }^{52}$ Wallén, P., Carlsson, K., Liljeborg, A. and Grillner, S. (1988) Three-dimensional reconstruction of neurons in the lamprey spinal cord in whole-mount, using a confocal laser scanning microscope. Journal of Neuroscience Methods 24, 91-100.

${ }^{53}$ Micheva, K. D. and Smith, S. J. (2007) Array tomography: a new tool for imaging the molecular architecture and ultrastructure of neural circuits. Neuron 55 (1), 25-36.

${ }^{54}$ Prior, F. W., Bushey, R. M., Milner R. J. and Larson-Prior, L. J. (1993) Automated segmentation of cerebellar Purkinje cell dendrites. J. Neurosci S19(Suppl.).
} 
segmentation of Purkinje cell dendrites, imaged in 3D by a Zeiss LSM and edge-enhanced by preprocessing.

An important characteristic of optical section data, including confocal, has been that the sampling resolution in depth generally has not equalled that within the image plane (although this is markedly improved through the use of multi-photon microscopy). Hence it often has been worthwhile to take the computationally intensive extra step of deconvolving 3D scans against optical point-spreadfunctions before attempting to segment the data (see a review by James G. McNally, from 1999) ${ }^{55}$.

One such segmentation method employs "seeding" (or "flood-filling") operations which accrete subsets of voxels that are recursively discovered to be adjacent. When objects in a $3 \mathrm{D}$ data set have high enough signal to noise, then very usable connected structures often can be obtained by flooding using simple intensity or gradient thresholds (see Figure 2 in Alfredo Rodriguez from $2003^{56}$, using the VoxelView software from Vital Images, founded in 1989 by Vincent J. Argiro). In instances where geometrically sensible sets of connected voxels are obtained, it then can be useful to subject the data to thinning ("skeletonizing") or watershed procedures, with the aim of obtaining the medial axis (roughly, the local center-point of the data). This can produce (for neurons) a tree structure which is one voxel thick, except at branch points, and that can be automatically traversed in order to extract topological and geometrical properties (see the 1995 work of Mark F. Villa and Franklin R. Amthor ${ }^{57}$ for the $2 \mathrm{D}$ and Stephen L. Senft ${ }^{58}$ for the 3D case). It also is possible in this way to recover an estimate of the diameter at each point, an important variable for understanding the potential metabolic and electrochemical properties of cells.

Unfortunately, scanned objects of interest frequently contain gaps, or are obscured by noise or other unwanted signals. Overcoming such defects may require little effort for human sight, but for the computer even complex heuristic procedures tend to only address the issue for a limited variety of image textures. Andrew R. Cohen, et al. ${ }^{59}$

\footnotetext{
$\overline{55}$ McNally, J. G., Karpova, T., Cooper, J. and Conchello, J. A. (1999) Three-Dimensional Imaging by Deconvolution Microscopy. Methods 19, 373-385.

${ }^{56}$ Rodriguez, A., Ehlenberger, D., Kelliher, K., Einstein, M., Henderson, S. C., Morrison, J. H., Hof, P. R., and Wearne, S. L. (2003) Automated reconstruction of three-dimensional neuronal morphology from laser scanning microscopy images. Methods 30, 94-105

${ }^{57}$ Villa, M.F. and Amthor, F.R. (1995) Automating the quantitative analysis of 2-D neural dendritic trees. J. Neurosci. Meth. 56, 77-88.

${ }^{58}$ Senft, S. L. (1995) Derivation of neuron geometry from confocal scans. J. Neurosci . S21(Suppl.), 1078.

${ }^{59}$ Cohen, A.R., Roysam, B. \& Turner, J.N. (1994) Automated tracing and volume measurements of neurons from 3-D confocal fluorescence microscopy data J. Microsc. 173 (part 2), 103-114.
}

were able to automatically reconstruct pyramidal neurons (apart from very fine processes) in 1994, using a point-process-based initial segmentation that provided filtered input to subsequent seeding and thinning steps (similar to the approaches mentioned above). Later efforts in 2002 by Khalid A. Al-Kofahi ${ }^{60}$ in the same group circumvented the need for flood-filling and skeletonizing by employing tubular snake-like primitives in a combined edge-detection and tree-traversal process. Reconstructions from multiple overlapping deconvolved 3D confocal stacks were semi-automatically obtained by Rodriguez et al. in 2003. As with computerized microscope development decades earlier, in the early 2000 s some algorithmic methods began to be commercialized. Examples include AutoNeuron (from MicroBrightfield), and FilamentTracer (previously NeuronTracer, from BitPlane, founded by Marius Messerli). For a recent survey of reconstruction tools, see Erik Meijering's review of $2010^{61}$.

Technical refinements in this field are still needed in order to better follow discontinuous processes - given the great range in staining methods - and to disambiguate topological peculiarities which can be confusing even to human operators. Also, the representation of somata could be systematically improved (for helpful insight, see Haijime Mannen ${ }^{62}$, or P. N. Gaunt and Walter Alwyn Gaunt ${ }^{63}$ ). Although spine morphology can now been measured reliably (for instance as shown in 2009 by Qing $\mathrm{Li}$, et $\mathrm{al}^{64}$ ), that additional type of detail by no means exhausts the natural variation of cell shape. Tree-like cylindrical abstractions suffice to describe many canonical vertebrate neurons, such as bipolar, stellate, pyramidal, Purkinje and so on, but it is helpful to recall that some glia are intricately lamellar entities, and that invertebrates partition functionality differently between their dendrites and axons (which can exhibit markedly non circular, often invaginated, cross sections).

Some of these challenging issues are becoming addressable by the latest wave of methods reported in

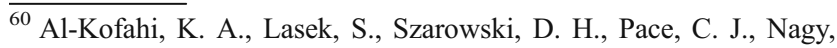
G., Turner, J. N., and Roysam, B. (2002). Rapid automated threedimensional tracing of neurons from confocal image stacks. IEEE Trans. Information Technology in Biomedicine, 6 (2), 171.

${ }^{61}$ Meijering, E. (2010) Neuron Tracing in Perspective. Cytometry 77A (7), 693-704.

${ }^{62}$ Mannen, H. (1975) Morphological analysis of an individual neuron with Golgi's method. In: M. Santini, Editor, Golgi Centennial Symposium Proceedings, Raven Press, New York.

${ }^{63}$ Gaunt, P. N. and Gaunt, W. A. (1978) Three dimensional reconstruction in biology. Pitman Medical Publishing, Kent.

${ }^{64}$ Li, Q., Zhou, X., Deng, Z., Baron, M., Teylan, M. A., Yong, K., Wong, S. T. C. (2009) A novel surface-based geometric approach for 3D dendritic spine detection from multi-photon excitation microscopy images. Proc IEEE Int Symp Biomed Imaging. From Nano to Macro, $1255-1258$.
} 
this issue $65,66,67,68,69,70$. However, the ensemble of anatomical variation seen in nature eventually may require more flexible data structures than currently in use, in order to address the plethora of shapes in excitable tissue. This need may become particularly acute as very high resolution photonic imaging begins to generate a level of detail historically the purview of electron microscopy, and as 3D reconstruction methods for EM continue to mature (such as the 2009 work of Angel Merchán-Pérez) ${ }^{71}$.

Besides surface geometry, numerous other sub-cellular and extra-cellular localizable features also are germane to an understanding of the functioning nervous system. Chemical synapses are the most focal and well-known conduits for neuronal communication, and spines are prominent as potential synaptic targets, as well as delimiters of local processing compartments. Consequently synaptic elements now can be accommodated in reconstructed data structures. But strategically located gap junctions are likewise important both for cell-cell synchrony and for material transferparticularly in the immature nervous system. In addition, the three-dimensional distribution of mitochondria is becoming pertinent to an understanding of network capacity to resist fatigue, and data structures that represent branched neurons will have to be capable of accommodating these and other categories of physiological detail.

For these sorts of reasons, it will become increasingly important to reconstruct multiple interacting cells, and their interiors. DIADEM's scope thus naturally extends to network reconstruction - both sparse and dense-and in the latter case methods will be needed to blend tree reconstructions with volumetric reconstruction of the tissue plenum, as perennially desired for electron microscopy.

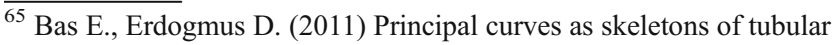
objects: Locally characterizing the structures of axons. Neuroinformatics. doi:10.1007/s12021-011-9105-2

${ }^{66}$ Chothani P., Mehta V., Stepanyants A. (2011) Automated tracing of neurites from light microscopy stacks of images. Neuroinformatics. doi:10.1007/s12021-011-9121-2.

${ }^{67}$ Türetken E., Gonzalez G., Blum C., Fua P. (2011) Automated reconstruction of dendritic and axonal trees by global optimization with geometric priors. Neuroinformatics. doi:10.1007/s12021-0119122-1.

${ }^{68}$ Zhao T., Xie J., Amat F., Clack N., Ahammad P., Peng H., Long F., et al., (2011) Automated reconstruction of neuronal morphology based on local geometrical and global structural models. Neuroinformatics. doi:10.1007/s12021-011-9120-3.

${ }^{69}$ Wang Y., Narayanaswamy A., Tsai C.-L., Roysam B. (2011) A broadly applicable 3-D neuron tracing method based on open-curve snake. Neuroinformatics. doi:10.1007/s12021-011-9110-5.

${ }^{70}$ Narayanaswamy A., Wang Y., Roysam B. (2011) 3-D image preprocessing algorithms for improved automated tracing of neuronal arbors. Neuroinformatics. doi:10.1007/s12021-011-9116-z.

${ }^{71}$ Merchán-Pérez, A., Rodriguez, J-R., Alonso-Nanclares, L., Schertel, A. and DeFelipe, J. (2009) Counting synapses using FIB/SEM microscopy: a true revolution for ultrastructural volume reconstruction. Frontiers in Neuroanatomy 3, 1-14.
}

Having a single method able to segment both light and EM data would be extremely helpful, and for that purpose deformable, branching, space-filling automata (and that perhaps emulate some aspects of growth cone navigation as they meander through $3 \mathrm{D}$ data sets, as a predictive aid to reconstruction) might initially fill the bill.

This has been a staccato history of the discovery and 3D documentation of single neurons. Because of constraints in knowledge, time and space the important efforts of many have be omitted, and there may be inaccuracies (since of course relatively little of it could be first-hand experience).

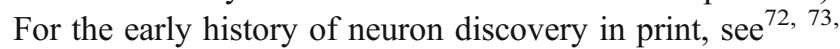
$74,75,76$. There are also numerous internet resources, including $77,78,79,80$. The reader should also understand that encompassing this narrow retrospective there is a vastly more extensive intellectual history of the discoveries of brain tissue (glia, blood vessels, CSF), brain regions, and brain physiology. One will eventually wish for any nearterm analytic and synthetic computational successes spurred by DIADEM's current focus on reconstructing single cells or small cell groups to be informed by insights and techniques from that larger realm and to be enormously extended.

Peering into one possible future aspect of brain reconstruction, it would be fascinating to precisely reconstruct arrays of exemplar local networks and pockets of intricately entwined neuropil. That could enable one to begin to determine which abstract subregions within the enormous space of possible geometric configurations and range of possible functional transformations have actually been instantiated through evolution. As more numerous neural structures become reconstructed it should become possible to encapsulate their descriptions in rather precise statistical formats, which may differentiate subtilely among classes of neurons and their functional interconnections. In turn, having such a library of cell descriptions would make

\footnotetext{
${ }^{72}$ Clarke, E. and O'Malley, C. D. (1968) The human brain and spinal cord: a historical study illustrated by writings from Antiquity to the Twentieth Century. University of California Press.

${ }^{73}$ Finger, S. (1994) Origins of neuroscience: a history of explorations into brain function. New York, Oxford University Press.

${ }^{74}$ Schmahmann, J. D. and Pandya, D. N. (2006) Fiber pathways of the brain. Oxford University Press, USA.

${ }^{75}$ Whitaker, H. A., Smith, C. U. M., and Finger, S. (2007) Brain, mind and medicine: essays in eighteenth-century neuroscience. Springer, New York.

${ }^{76}$ William Turner (1875) History of Anatomy. Encyclopedia Britannica 9th ed. v. 1 pp. $799-819$.

${ }^{77} \mathrm{http}: / /$ faculty.washington.edu/chudler/hist.html

$78 \mathrm{http}: / /$ www.victorianmicroscopeslides.com/slides.htm

${ }^{79} \mathrm{http}: / /$ www.cerebromente.org.br/n17/history/neurons2_i.htm

${ }^{80} \mathrm{http}: / /$ scienceblogs.com/neurophilosophy/2007/07/the_discovery_of the neuron.php
} 
feasible the emulation of large regions of CNS, by means of algorithmically generated arbors that project to and functionally connect with those of target cells. Generated networks of this sort have the possibility of being further defined in their spatial location by the progressively refined 3D brain Atlases that are being created for many species.
Being based on the statistics of accurate reconstructions, this might be the best strategy (in the interim until the actual anatomy of vast areas of densely characterized CNS becomes available - which seems remote) for obtaining tangible and realistic representations of brain organization that are both highly detailed and can coherently span large areas. 